in Knowledge of mothers about breast feeding, $42.5 \%$ had good knowledge, $56.5 \%$ had average knowledge.

Conclusion Raise awareness of breast-feeding mothers than exclusively breastfeeding, were the important health priorities in the health of children.

feeding, breastfeeding, attitudes, mothers, infants

\section{DILATATIVE CARDIOMYOPATHY - CASE REPORT}

doi:10.1136/archdischild-2012-302724.0500

MV Sofranac. Paediatric, Health Centre Danilovgrad, Danilovgrad, Montenegro

Background and Aims Dilatative cardiomyopathy/DC/is a myocardium disease characterized by increased dimension of heart cavites and general weakening of the systolic function/the most frequently of the left ventricle/, with emersion ofsymptoms and signs of a cardiac insufficiency. Etiologically, there are family and genetic factors. Manifestations of the disease at infants are feeding problems, difficult and accelerated breathing and excessive sweating. Older children complain of fatigue, difficult breathing and hacking. It can olso be asymptomatic and discovered at occasional medical examination with an x-ray finding of cardiomegaly or electrocardiogram changes, or with an appearance of an unspecific symptom, as was he case here.

Methods Data analysis of the case history of the patient with DC. Anamnestic data, laboratory analysis and data through clinical diagnostic procedures of reference institutions are used as work methods. Purpose is to show the patient with a diagnosed DC with an initial unspecific symptom.

Results The work show a boy old 14 with dyspnea as the only symptom. Forth child of the fifth normal pregnancy/death of the two-month old sister caused by a heart condition of unknown etiology/. Slowed development as infant, frequent respiratory infections. A boy adipose, tachypneic, dyspneic. Sistolic murmur at apex. TA100/60mmHg, CP100/min, sO290\%.X-ray:cardiomegaly. In competent institution diagnosis is confirmed.

Conclusions Initial clinical presentation of the disease at patients with DC can be characterized by unspecific respiratory disturbances, as was the case with this patient. Echocardiography remains a sovereign method in establishing the diagnosis. In the future, endomyocardial biopsy is expected to offer decisive data regarding the etiopathogenesis of this condition which would enable a timely causal medication therapy and avoid surgical therapy.

\section{THE INFLUENCE OF BETA - BLOCKER (BISOPROLOL) ON HEART RATE VARIABILITY IN CHILDREN WITH MITRAL VALVE PROLAPSE}

doi:10.1136/archdischild-2012-302724.0501

'L Romanciuc, ${ }^{2,3} \mathrm{~N}$ Revenco. 'Pediatrics Department, State Medical and Pharmaceutical University 'N. Testemitanu'; 'Pediatrics Department; ${ }^{3}$ Research Institute for Maternal and Child Healthcare, Chisinau, Moldova

The carried out research has included 50 children: I group (bisoprolol)- $60 \%$ children and II group (placebo) - 40.0\%, average age (13.5 \pm 0.60 ) years; doses of bisoprolol (PO): $1.25-2.5 \mathrm{mg} / \mathrm{kg} / \mathrm{dose}$.

ECG monitoring has defined heart rate average maximum and minimum value in the beginning of research and in dynamics a month later. Dynamics in a month of action of a bisoprolol has not changed the minimum values of heart rate in the first group in comparison with placebo ( $p>0.05)$, but has lowered the maximum values of heart rate reductions at children with MVP $(-8.74 ; p<0.01)$ in the first group in comparison with placebo $(0.55 ; p>0.05)$ and average values of heart rate $(-4.70 ; p<0,001)$ in the first group in comparison with placebo $(-0.10 ; p>0.05)$. Statistical parametres HRV (SDNN and PNN 50) defined in the beginning in both groups did not differ considerably $(\mathrm{p}>0.05)$. Dynamics in a month of action bisoprolol on indicators HRV was showed by decrease in value PNN 50 in the first group $(-6.42 ; \mathrm{p}<0,001)$.

Results of research have revealed, that at children with MVP, bisoprolol has considerably reduced heart rate $(-8.9$; $p<0,001)$ in comparison with placebo, without influence on systolic and diastolic blood pressure. Influence bisoprolol on indicators HRV in the first group it was characterised by decrease of statistics PNN 50 characteristic for activity of sympathetic vegetative nervous system $(-6.42 ; \mathrm{p}<0,001)$.

\section{EXPLAINING THE RELATION OF SOCIOECONOMIC STATUS TO CHILDHOOD BLOOD PRESSURE. THE ABCD-STUDY}

doi:10.1136/archdischild-2012-302724.0502

${ }^{1,2}{ }^{2} \mathrm{G}$ van den Berg, ${ }^{2} \mathrm{M}$ van Eijsden, ${ }^{3} \mathrm{TGM}$ Vrijkotte, ${ }^{1} \mathrm{RJBJ}$ Gemke. ${ }^{1}$ Pediatrics, VU University Medical Center; ${ }^{2}$ Epidemiology, Documentation and Health Promotion, Public Health Service of Amsterdam; ${ }^{3}$ Public Health, Academic Medical Center, University of Amsterdam, Amsterdam, The Netherlands

Objective We investigated the association of socioeconomic status to blood pressure and prehypertension in childhood.

Methods In a prospective cohort study (ABCD-study) we obtained blood pressure measurements and information on potential explaining factors, namely birth weight, breastfeeding duration, and body mass index (BMI) in 3067 children of 5-6 year of age.

Results The systolic- and diastolic blood pressures of children from mid-educated women were $1.0 \mathrm{~mm} \mathrm{Hg}$ higher (95\% CI 0.4-1.7) and $0.9 \mathrm{~mm} \mathrm{Hg}$ higher (95\% CI 0.3-1.4), and the blood pressures of children from low-educated women were $2.2 \mathrm{~mm} \mathrm{Hg}$ higher $(95 \% \mathrm{CI}$ 1.4-3.0) and $1.7 \mathrm{~mm} \mathrm{Hg}$ higher (95\% CI 1.1-2.4), compared to children from high-educated women (models controlled for age, gender, height, and ethnicity). Children of mid- or low-educated mothers were also more likely to have prehypertension (>p90;21\% and $27 \%$ ) compared to children of high educated mothers (13\%). In addition, these associations could partly be explained by birth weight, breastfeeding duration, and BMI, but remained significant following adjustment for these variables. Income adequacy was less clearly associated with prehypertension, even after including potential mediators.

Conclusion The socio-economic status related differences in blood pressure seem to emerge from childhood as the results show a higher blood pressure and more prehypertension in children from lower SES. Improving birth weight, breastfeeding duration, and BMI, might help decreasing the socio-economic disparities, but other factors might also play a role.

\section{SEPARATING THE ASSOCIATIONS OF PROGRAMMING AND TRACKING DURING INFANCY WITH CHILDHOOD BLOOD PRESSURE, THE ABCD STUDY}

doi:10.1136/archdischild-2012-302724.0503

${ }^{1} \mathrm{M}$ de Beer, ${ }^{2} \mathrm{M}$ van Eijsden, ${ }^{3} \mathrm{~T}$ Vrijkotte, ${ }^{4} \mathrm{C}$ Fall, ${ }^{4} \mathrm{C}$ Osmond, ${ }^{1} \mathrm{R}$ Gemke. ${ }^{\text {PPediatrics, }}$ VU University Medical Center; ${ }^{2}$ Epidemiology, Documentation and Health Promotion, Municipal Health Service; ${ }^{3}$ Social Medicine, Academic Medical Center, Amsterdam, The Netherlands; ${ }^{4}$ MRC Lifecourse Epidemiology Unit, Southampton General Hospital, Southampton, UK

Background and Aims In a prospective pregnancy cohort study, we investigated the association of early growth with blood pressure at 5-6 years of age.

Methods Our study was based on 2,338 children who were born healthy at $\geq 37$ completed weeks gestation with on average 7 measures of growth (weight and length) from birth to age 12 months. We used conditional weight, a residual of current weight regressed on prior weights, to represent deviations from expected weight gain from 0 to 1,1 to 3,3 to 6 , and 6 to 12 months. The same method 
was applied for conditional length and body mass index. Associations of these measures with systolic and diastolic blood pressure at the age of 5-6 years were modeled before and after adjustment for current body mass index and height.

Results Higher weight-, length-, and bmi conditionals (expressing faster growth) were associated with higher blood pressure, with the exception of conditional growth between birth and 1 month. Adjusted for current height and body mass index, almost none of the conditionals were associated with blood pressure. However, lower birth weight and lower weight and height conditionals 0 to 1 month were associated with higher systolic blood pressure at age 5-6 years.

Conclusions We infer that the inverse association between prenatal and first month growth with childhood blood pressure may reflect a programming effect, while the positive association between infant growth after 1 month with childhood blood pressure probably reflects an effect of the tracking of body size.

\section{AETIOLOGY AND ELECTROCARDIOGRAM FINDINGS IN CHILDREN PRESENTING TO THE EMERGENCY DEPARTMENT WITH CHEST PAIN}

doi:10.1136/archdischild-2012-302724.0504

'RHK Tan, ${ }^{2} \mathrm{R}$ Saravanan, ${ }^{2} \mathrm{E}$ Ladusans, ${ }^{1} \mathrm{M}$ Ryan. ${ }^{1}$ Accident \& Emergency Department; ${ }^{2}$ Cardiology Department, Alder Hey Childrens' Hospital, Liverpool, UK

Introduction Chest pain is a frequent cause of attendance to the emergency department. Electrocardiogram (ECG) is often used as a first line investigation. This study aims to investigate the aetiology of chest pain and usefulness of ECG in a group of 200 children ranging between 3-16 years who presented between June - December 2009.

Methods ED casenotes were reviewed retrospectively to study the demographic features, clinical characteristics, ECG utilization and final diagnoses of these children. Children were placed in two groups. The first group were those who had suggestive history (crushing/exertional pain/palpitations), positive examination findings, or previous cardiac/family history. The second group of patients consisted of those without these features.

Results Musculoskeletal chest pain (46\%) is the most common diagnosis in children followed by respiratory $(15 \%)$, gastrointestinal $(14 \%)$, idiopathic $(13 \%)$, and psychological $(7.5 \%)$ causes. Cardiac cause chest pain $(4.5 \%)$ was the least common.

Only 20 patients $(10 \%)$ presented with positive cardiac features according to the criteria used. 81 (40.5\%) patients in total had an ECG performed. Of the 20 patients with cardiac features, $15(75 \%)$ had an ECG compared to $66(37 \%)$ patients in the other group. $(p<0.0014)$ Four abnormal ECGs were obtained. These were all from the group with positive cardiac features. $(p<0.0008)$.

Conclusion Musculoskeletal pain was found to be the most common cause of chest pain. Electrocardiogram has poor yield when performed in children without significant cardiac history/examination findings. However, it remains a useful screening tool and is beneficial for reassurance of patients and their families.

\section{LINEAR GROWTH IN CHILDREN WITH CONGENITAL ACYANOTIC HEART DISEASE (CHD) BEFORE VERSUS AFTER SURGICAL INTERVENTION}

doi:10.1136/archdischild-2012-302724.0505

${ }^{1} \mathrm{~A}$ Soliman, ${ }^{2} \mathrm{H}$ Yassin, ${ }^{2} \mathrm{~S}$ Saeed, ${ }^{2} \mathrm{~A}$ Khellah, ${ }^{3 \mathrm{R}}$ Elalaily, ${ }^{1} \mathrm{~A}$ Elawwa. 'Pediatrics; ${ }^{2}$ Cardiology; ${ }^{3}$ Primary Health Care, Hamad Medical Center, Doha, Oatar

Objectives To evaluate linear growth and insulin-like growth factor- I (IGF-I) concentration of patients with congenital acyanotic heart disease (CHD) before versus after surgical intervention.
Design 27 infants and children with CHD (10 with VSD, 8 with ASD, 9 PDA) without heart failure, before and 12 months or more after surgical or catheter intervention. Eighty normal children served as controls.

Results At presentation, age $=(35.6+/-26$ months $)$, patients were significantly shorter, height SDS $(\mathrm{HtSDS})(-1.6+/-1.1)$ and had lower BMI $(15.1+/-2.5)$ compared to normal controls (HtSDS = $0.25+/-0.3, \mathrm{BMI}=(16.4+/-1.5)$. One year or more after catheter or surgical treatment the HtSDS and BMI increased significantly in patients to $-0.55+/-0.9$ and $15.9+/-1.5$ respectively. Circulating concentrations of IGF-I increased from $46.8+/-29 \mathrm{mcg} / \mathrm{L}$ before to $77.3+/-47.6 \mathrm{mcg} / \mathrm{L}$ after intervention. The HtSDS after treatment was correlated with the IGF-I concentration $(r=0.804, \mathrm{P}<0.001)$. The change in the HtSDS after intervention was correlated significantly with BMI $(r=0.594, p<0.001)$. The shunt size was correlated negatively with BMI before intervention $(r=-0.35, \mathrm{P}<0.01)$ and with HtSDS $(r=-0.461, p<0.05)$.

Conclusions These data denoted that early surgical interference and good weight gain have beneficial effect on postoperative growth spurt. The accelerated linear growth after intervention appears to be mediated through activation of the GH/IGF-I system.

\section{DO SOUTH ASIAN BABIES HAVE MORE CONGENITAL HEART DEFECTS, DOES CONSANGUINITY INFLUENCE IT? FINDINGS FROM BORN IN BRADFORD STUDY}

doi:10.1136/archdischild-2012-302724.0506

'P Agadoorappa, ${ }^{2}$ S Oddie, ${ }^{3} \mathrm{~J} \mathrm{Gibbs,}{ }^{4} \mathrm{~N}$ Pawson, Born in Bradford Cohort. 'Paediatrics, Bradford Teaching Hospitals, Leeds; 'Neonatology, Bradford Teaching Hospitals, Bradford; ${ }^{3}$ Paediatric Cardiology, Leeds Teaching Hospitals NHS Trust, Leeds; ${ }^{4}$ Bradford Institute of Health Research, Bradford Teaching Hospitals, Bradford, UK

Background and Aims Born in Bradford is a birth cohort study established in March 2007 to look at health and outcomes in a multi ethnic population in Bradford (UK). We aimed to establish whether South Asian children in the cohort had a higher prevalence of congenital cardiac anomalies compared to non South Asians, and evaluate association with consanguinity.

Methods Babies with cardiac anomalies in the cohort born between May 2007 and March 2010 identified using multiple ascertainment models. Ethnicity ascribed to cases using clinical records $\&$ inferred to cohort based on questionnaire responses. Data on consanguinity and other cofounders were obtained from database. Statistical analysis done by Chi square test and logistic regression.

Results Structural congenital cardiac defects $=96$ cases. Overall prevalence rate $=8.8 / 1000$ live births. Prevalence was significantly higher in South Asians compared to non South Asians for all cardiac anomalies $(11 / 1000$ vs $6.2 / 1000, \mathrm{P}<0.05) \&$ anomalies including only VSDs requiring surgery $(8.7 / 1000$ vs $4.4 / 1000, \mathrm{P}<0.05)$ implying that the differences were unlikely due to ascertainment bias. Complex cardiac defects were more prevalent in South Asians, cyanotic defects were significantly higher in this group $\mathrm{P}=0.017$. South Asian ethnicity (Odds-ratio=1.76, $\mathrm{P}=0.019$ ) and consanguinity (Odds-ratio $=2.002, \mathrm{P}=0.003$ ) significantly increased the risk for cardiac anomalies in univariate analysis. Multivariate analysis incorporating confounding factors attenuated their effect; however consanguinity remained a borderline significant predictor.

Conclusions We have demonstrated an apparent excess of congenital cardiac anomalies in South Asians in the Born in Bradford cohort. Consanguinity seemed to increase the risk for cardiac anomalies.

\section{A NOVEL METHOD FOR QUANTIFICATION OF LEFT VENTRICULAR HYPERTRABECULATION/NONCOMPACTION USING TWO-DIMENSIONAL ECHOCARDIOGRAPHY}

doi:10.1136/archdischild-2012-302724.0507 